\title{
OPEN Strain effects on polycrystalline germanium thin films
}

\author{
Toshifumi Imajo ${ }^{1,2 \bowtie}$, Takashi Suemasu ${ }^{1} \&$ Kaoru Toko ${ }^{1,3 凶}$ \\ Polycrystalline Ge thin films have attracted increasing attention because their hole mobilities exceed \\ those of single-crystal Si wafers, while the process temperature is low. In this study, we investigate the \\ strain effects on the crystal and electrical properties of polycrystalline Ge layers formed by solid-phase \\ crystallization at $375^{\circ} \mathrm{C}$ by modulating the substrate material. The strain of the Ge layers is in the \\ range of approximately $0.5 \%$ (tensile) to $-0.5 \%$ (compressive), which reflects both thermal expansion \\ difference between $\mathrm{Ge}$ and substrate and phase transition of Ge from amorphous to crystalline. \\ For both tensile and compressive strains, a large strain provides large crystal grains with sizes of \\ approximately $10 \mu \mathrm{m}$ owing to growth promotion. The potential barrier height of the grain boundary \\ strongly depends on the strain and its direction. It is increased by tensile strain and decreased by \\ compressive strain. These findings will be useful for the design of Ge-based thin-film devices on various \\ materials for Internet-of-things technologies.
}

Internet-of-things (IoT) will largely increase the number of electronic devices. In this regard, it is crucial to provide electronic functions to various objects. Numerous general-purpose substrates, such as low-cost glass and plastic, have low heat resistance temperatures, which limits the process temperature of device fabrication. Ge is a promising electronic material for IoT devices because of its high carrier mobilities (electron: $3900 \mathrm{~cm}^{2} \mathrm{~V}^{-1} \mathrm{~s}^{-1}$, hole: $1900 \mathrm{~cm}^{2} \mathrm{~V}^{-1} \mathrm{~s}^{-1}$ ) and low crystallization temperature (approximately $\left.500{ }^{\circ} \mathrm{C}\right)^{1,2}$. The performances of metal-oxide-semiconductor field-effect transistors (MOSFETs) based on a single-crystal Ge-on-insulator structure, formed using a single-crystal wafer ${ }^{3-5}$ and/or high-temperature process $\left(>900^{\circ} \mathrm{C}\right)^{6,7}$, surpassed those of Si MOSFETs. Low-temperature syntheses of Ge thin-film transistors (TFTs) have been achieved using conventional solid-phase crystallization $(\mathrm{SPC})^{8-11}$, laser annealing ${ }^{12}$, seed layer technique ${ }^{13}$, and metal-induced crystallization $^{14-16}$. Although these methods are potentially useful to expand the application of Ge, the crystallinity of the resulting polycrystalline Ge (poly-Ge) is still insufficient to increase the performances of Ge TFTs over those of Si MOSFETs.

Among the synthesis methods for Ge, SPC has been considerably advanced in recent years, which provided an increased grain size of poly-Ge by heat deposition of amorphous $\mathrm{Ge}(\mathrm{a}-\mathrm{Ge})$ precursors ${ }^{17}$ and addition of $\mathrm{Sn}$ in $\mathrm{Ge}^{18-20}$. Accumulation-mode $p$-channel transistors based on the $p$-type Ge exhibited field-effect mobilities (170 $\mathrm{cm}^{2} \mathrm{~V}^{-1} \mathrm{~s}^{-1}$ ) higher than those of most $p$-channel Si transistors ${ }^{21}$. The formation of a $\mathrm{GeO}_{x}$ underlayer further improved the Hall hole mobility of poly-Ge to $620 \mathrm{~cm}^{2} \mathrm{~V}^{-1} \mathrm{~s}^{-122}$. Although poly-Ge is generally $p$-type owing to the high hole concentration derived from defect-induced acceptors ${ }^{23,24}, n$-type poly-Ge layers with high electron mobilities have also been achived ${ }^{25,26}$.

Numerical studies have been carried out on the effects of strain in single-crystal $\mathrm{Ge}^{27}$, because the strain in Ge contributes to a carrier mobility increase and direct transition change ${ }^{28}$. Generally, poly-Ge layers have small tensile strains, although the origin of the strain has not been completely understood ${ }^{9}$. The strain naturally introduced in poly-Ge layers is not sufficiently large to affect the basic physical properties of Ge. However, the strain may affect the crystal growth and grain boundary (GB) properties of poly-Ge layers, which has not been extensively investigated. In this study, we control the strains in high-mobility poly-Ge layers formed by a lowtemperature SPC using the difference in thermal expansion with respect to the substrate. The magnitude and direction of the strain largely influence the grain size and GB barrier height of the poly-Ge layers.

\section{Experimental}

To modulate the strain applied to the Ge layer, we used various substrates with different coefficients of thermal expansion (CTEs), including amorphous $\mathrm{SiO}_{2}$, polyimide (PI), single-crystal Si (001), Ge (110), $\mathrm{SrTiO}_{3}(\mathrm{STO})$ (001), and $\mathrm{CaF}_{2}$ (001). We denote the CTE difference between $\mathrm{Ge}$ and substrate as $\Delta \alpha$; the values are presented in

${ }^{1}$ Institute of Applied Physics, University of Tsukuba, 1-1-1 Tennodai, Tsukuba, Ibaraki 305-8573, Japan. ${ }^{2}$ JSPS Research Fellow, 8 Ichiban-cho, Chiyoda-ku, Tokyo 102-8472, Japan. ${ }^{3}$ PRESTO, Japan Science and Technology

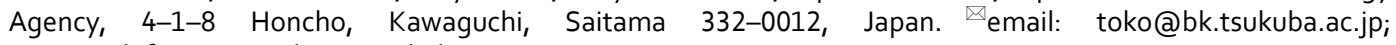
imajo.toshifumi.mg@alumni.tsukuba.ac.jp 


\begin{tabular}{|l|l|l|}
\hline Substrate & $\mathbf{C T E}\left[\mathbf{1 0}^{\mathbf{- 6}} \mathbf{K}^{-\mathbf{1}}\right]$ & $\mathbf{\Delta} \boldsymbol{\alpha}\left[\mathbf{1 0}^{-\mathbf{6}} \mathbf{K}^{-\mathbf{1}}\right]$ \\
\hline $\mathrm{SiO}_{2}$ & 0.5 & -5.3 \\
\hline $\mathrm{Si}$ & 3.9 & -1.9 \\
\hline $\mathrm{Ge}$ & 5.8 & 0 \\
\hline $\mathrm{STO}$ & 9.4 & 3.6 \\
\hline $\mathrm{CaF}_{2}$ & 18.9 & 13.1 \\
\hline $\mathrm{PI}$ & 27.0 & 21.2 \\
\hline
\end{tabular}

Table 1. CTEs and CTE differences between Ge and substrate $(\Delta \alpha)$.
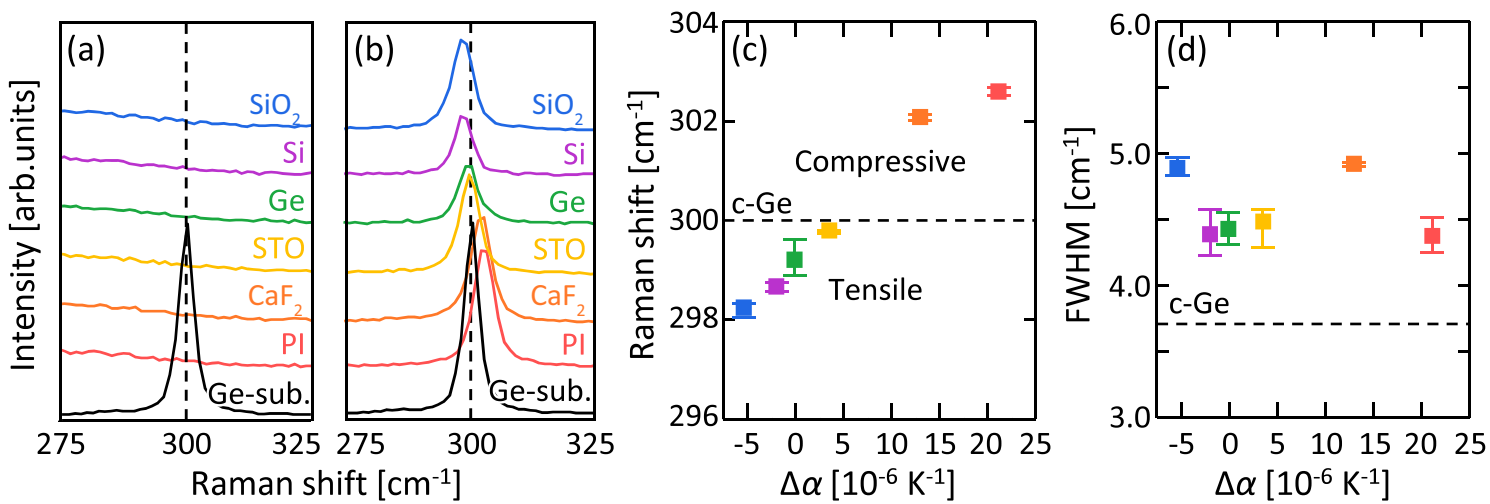

Figure 1. Raman spectroscopy analysis of the Ge layers on various substrates. (a, b) Raman spectra of the samples (a) before and (b) after annealing; the spectrum of a single-crystal Ge (100) wafer is shown for comparison. (c) Raman shifts and (d) FWHMs of the Ge peaks as functions of $\Delta \alpha$; the values for the Ge wafer are shown by the dotted lines. The error bars indicate the variability in the Raman measurements at five locations for each 1-cm square sample.

Table 1. Before the thin film deposition, the substrates were cleaned with acetone, methanol, and distilled water. To eliminate the difference in the interface between $\mathrm{Ge}$ and substrate, we fabricated 50 -nm-thick $\mathrm{GeO}_{x}$ layers on the substrates using radio-frequency (RF) magnetron sputtering (base pressure: $3.0 \times 10^{-4} \mathrm{~Pa}$ ) with $10 \mathrm{sccm}$ Ar plasma (working pressure: $0.5 \mathrm{~Pa}$ ), where the RF power was set to $50 \mathrm{~W}$. The sample stage was not heated or rotated. The samples were then air-transferred from the sputtering chamber to a molecular beam deposition system (base pressure: $5 \times 10^{-7} \mathrm{~Pa}$ ) within five min to avoid the reaction of $\mathrm{GeO}_{x}$ with air. Subsequently, 400-nmthick a-Ge precursors were prepared using a Knudsen cell with a solid Ge source (purity: $99.999 \%$ ) at a deposition rate of $1 \mathrm{~nm} \mathrm{~min}{ }^{-1}$. During the deposition, the sample stage was heated at $150{ }^{\circ} \mathrm{C}$ to densify the a-Ge ${ }^{17}$. The samples were then loaded into a conventional tube furnace (Koyo Thermo Systems, KTF035N1) with $\mathrm{N}_{2}$ flow (purity: $99.9 \%$, flow rate: $0.1 \mathrm{~L} \mathrm{~min}^{-1}$ ) and annealed at $375^{\circ} \mathrm{C}$ for $150 \mathrm{~h}$ to induce SPC. The temperature was calibrated by placing a thermocouple directly on the tube furnace and was confirmed to be uniform within the sample stage.

The resulting samples were evaluated using the Raman spectroscopy, X-ray diffraction (XRD), electron backscatter diffraction (EBSD), and Hall effect measurement. Raman spectra were measured using a JASCO NRS5100 with a frequency doubled Nd:YAG laser (wavelength: $532 \mathrm{~nm}$, power: $0.5 \mathrm{~mW}$, spot diameter: $20 \mu \mathrm{m}$ ). The objective lens magnification was $20 \times$. The time of acquisition for each Raman spectrum was $60 \mathrm{~s} \times 2$. The absolute Raman shift (detector resolution: $0.42 \mathrm{~cm}^{-1}$ ) was calibrated using the transverse optical phonon line $\left(300 \mathrm{~cm}^{-1}\right)$ of single-crystal $\mathrm{Ge}(100)$. Out-of-plane $(\theta-2 \theta)$ and in-plane $\left(-2 \theta_{\chi}\right)$ XRD patterns were measured using a Rigaku SmartLab system with a Ge monochromator (wavelength: $1.54 \AA$ ) and a Cu Ka radiation source (voltage: $40 \mathrm{kV}$, current: $30 \mathrm{~mA}$ ). The scans ranged from $20^{\circ}$ to $60^{\circ}$ in $0.01^{\circ}$ steps. The EBSD analyses were performed using a JEOL JSM-7001F (voltage: $25 \mathrm{kV}$, current: $15 \mathrm{~mA}$ ) with a TSL OIM analysis attachment. The Hall effect measurement with the Van der Pauw method was performed using a Bio-Rad HL5500PC with a $3200 \mathrm{G}$ permanent magnet.

\section{Results and discussion}

Raman spectroscopy and XRD are used to determine the strains and crystallinities of the Ge layers after annealing. Figure 1(a) shows that the Raman spectra of the samples before annealing have no peaks, indicating the amorphous state of Ge. Figure 1(b) shows that the peak corresponding to the Ge-Ge vibration mode is located around $300 \mathrm{~cm}^{-1}$, which indicates that all samples are crystallized after the annealing. The Raman shift and full width at half maximum (FWHM) of the Ge-Ge peak was analyzed from the Raman spectra. Figure 1(c) show that the Raman shift increases with $\Delta \alpha$, indicating that the strain is tensile for $\Delta \alpha<5 \times 10^{-6} \mathrm{~K}^{-1}$ and compressive for $\Delta \alpha>5 \times 10^{-6} \mathrm{~K}^{-1}$. Figure $1(\mathrm{~d})$ show that the FWHM has no clear correlation with $\Delta \alpha$, suggesting that the Ge-Ge bond quality does not depend on $\Delta \alpha$. 

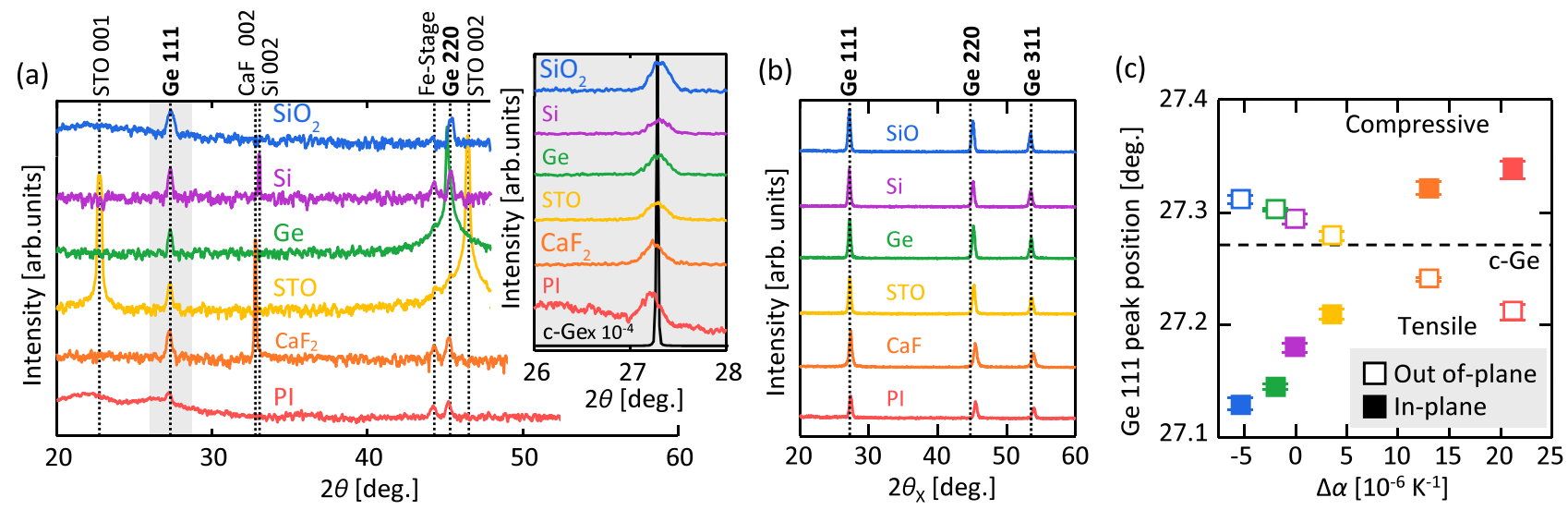

Figure 2. XRD analysis of the Ge layers on various substrates. (a) Out-of-plane $\theta-2 \theta$ XRD patterns; data for the bulk Ge (111) wafer are shown for comparison. The inset shows a magnified view focused on the Ge 111 peaks. (b) In-plane $\varphi-2 \theta_{\chi}$ XRD patterns. (c) Ge 111 peak position $\left(2 \theta\right.$ and $\left.2 \theta_{\chi}\right)$ as a function of $\Delta \alpha$; the value for the bulk Ge (111) wafer is shown by the dotted line. The error bars indicate the variability in the XRD measurements at four locations for each $1-\mathrm{cm}$ square sample.

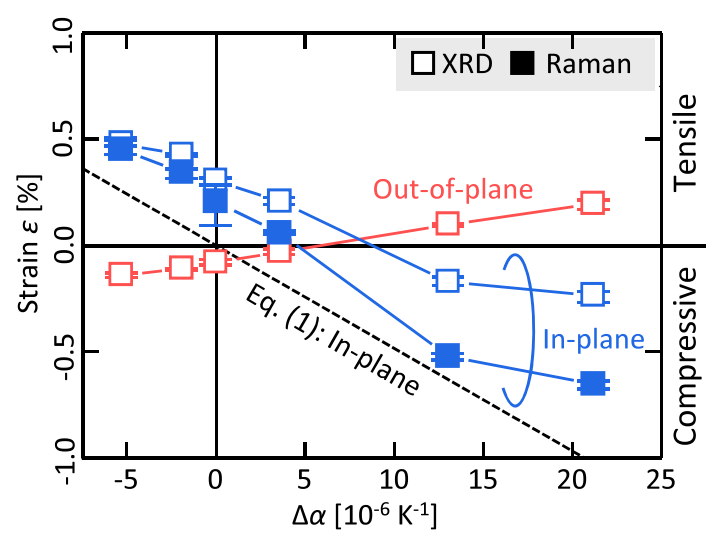

Figure 3. In-plane and out-of-plane strains $\varepsilon$ of the Ge layer as functions of $\Delta \alpha$. The experimental data were derived from the Raman (closed squares) and XRD (open squares) measurements. The theoretical data for the in-plane direction derived from Eq. (1) are shown by the dotted line. The error bars indicate the variability in the Raman and XRD measurements for each sample.

The out-of-plane $\theta-2 \theta$ XRD patterns in Fig. 2(a) show that all samples exhibit diffraction peaks corresponding to the Ge 111 and 220 planes, while other large peaks appear owing to the single-crystal substrates. The inset shows that the Ge 111 peak position is slightly shifted from that of a single-crystal Ge(111) wafer and varies depending on the substrate. The in-plane $\varphi-2 \theta_{\chi}$ XRD patterns in Fig. 2(b) show Ge 111, 220, and 311 peaks for all samples. According to the Ge powder diffraction patterns (Joint Committee on Powder Diffraction Standards (JCPDS) 04-0545), both XRD results indicate that the Ge layers are polycrystalline without epitaxial relationship with the substrates owing to the amorphous $\mathrm{GeO}_{x}$ interlayer. Figure 2(c) shows that the Ge 111 peak position of the Ge layer strongly depends on $\Delta \alpha$. The peak position increases with $\Delta \alpha$ in the in-plane measurement, while the opposite behavior is observed in the out-of-plane measurement. This behavior indicates that the directions of the strain in the Ge layer in the out-of-plane and in-plane measurements are opposite, which is consistent with the properties of general thin films.

Therefore, $\Delta \alpha$ affects the strain of the Ge layer. The strain corresponding to $\Delta \alpha$ should be applied to the Ge layer in the cooling process after the SPC. The strain $\varepsilon$ in the thin film caused by the thermal expansion difference is expressed by ${ }^{29}$

$$
\varepsilon=\frac{\Delta \alpha \cdot \Delta T}{1-v}
$$

where $\Delta T$ is the difference between the annealing temperature $\left(375^{\circ} \mathrm{C}\right)$ and room temperature $(300 \mathrm{~K})$ and $v$ is the Poisson's ratio of the thin film. The in-plane $\varepsilon$ in Ge attributed to the thermal expansion difference is theoretically obtained and presented by the dotted line in Fig. 3, which indicates that the Ge layer has a tensile strain for a negative $\Delta \alpha$ and compressive strain for a positive $\Delta \alpha$. We estimate $\varepsilon$ using the Raman shifts (Fig. 1(c)) of the Ge-Ge peak by the strain-phonon coefficient 
(a)

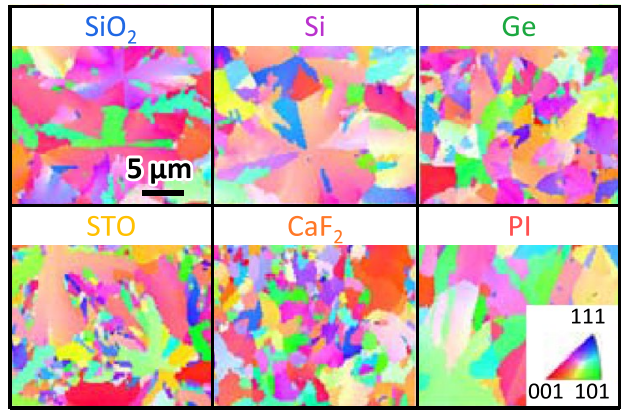

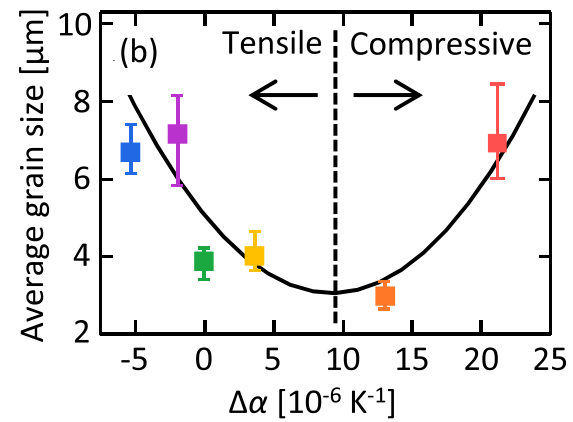

Figure 4. Grain sizes of the Ge layers on various substrates. (a) IPF images of the Ge layers, where the colors indicate the crystal orientations, according to the inset color scheme. (b) Average grain size determined by the EBSD analysis as a function of $\Delta \alpha$. The error bars indicate the variability in the EBSD measurements at four locations for each 1-cm square sample.

(a)

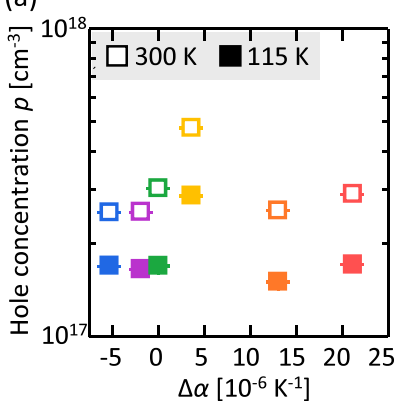

(b)

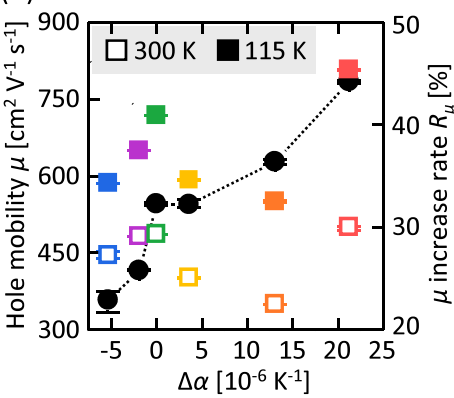

(c)

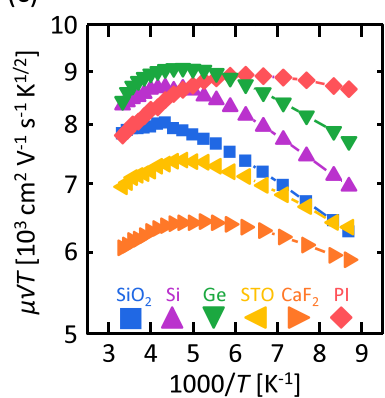

(d)

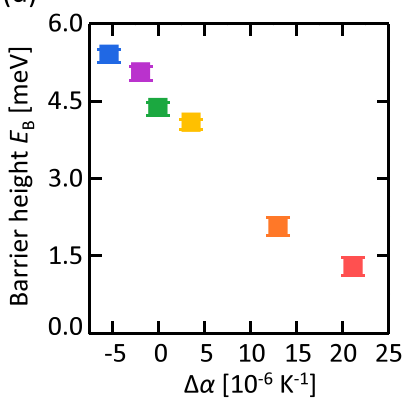

Figure 5. Electrical properties of the Ge layers. (a) Hole concentration $p$ and (b) hole mobility $\mu$ measured at $T=115 \mathrm{~K}$ and $300 \mathrm{~K}$ as functions of $\Delta \alpha$. The $\mu$ increase rate $R_{\mu}$ is also shown in (b). (c) Arrhenius plots of $\mu T^{1 / 2}$ for various substrates. (d) Grain boundary barrier height $E_{\mathrm{B}}$ as a function of $\Delta \alpha$. The error bars indicate the variability in the five measurements for each sample.

$\left(-395 \mathrm{~cm}^{-1}\right)$ proposed by Manganelli et al..$^{30}$ and Ge 111 peak positions in the XRD patterns (Fig. 2(c)) by the Bragg's law. The $\varepsilon$ values are different between the Raman and XRD analyses, which is likely because the strainphonon coefficient varies depending on the measurement environment and sample specifications ${ }^{30}$. Nevertheless, $\varepsilon$ obtained from the Raman and XRD analyses have similar tendency with respect to $\Delta \alpha$. The out-of-plane strain has a tendency opposite to that of the in-plane strain and exhibits smaller absolute $\varepsilon$ values, which is consistent with the Poisson effect. For the in-plane direction, the slopes of experimental $\varepsilon$ is almost consistent with the theoretical line derived from Eq. (1); however, the experimental $\varepsilon$ values shift above the theoretical $\varepsilon$ values for all $\Delta \alpha$. This suggests that the Ge layer is under a tensile stress regardless of the substrate. As known for the case of $\mathrm{Si}^{31}$, this occurs likely because the a-Ge tends to shrink during the crystallization owing to the density difference, while is constrained by the substrate. These results suggest that the total strain in the Ge layers is determined by not only the difference in thermal expansion with respect to the substrate, but also phase transition of Ge from amorphous to crystalline.

The grain size of the poly-Ge layer was evaluated using the EBSD analyses. The inverse pole figure (IPF) images in Fig. 4(a) show that all Ge layers consist of randomly oriented grains, indicating that there is no epitaxial relationship with the substrate, owing to the amorphous $\mathrm{GeO}_{x}$ underlayer. The grain sizes are large, on the order of micrometer, owing to the precursor densification and $\mathrm{GeO}_{x}$ underlayer effect ${ }^{22}$. The Ge grain size depends on the substrate species. Figure 4(b) shows a parabolic tendency with respect to $\Delta \alpha$. Considering the results of Fig. 3 together, Fig. 4(b) indicates that a larger $|\varepsilon|$ provides a larger grain size. During the heating of the sample for SPC, the a-Ge layer also has strain owing to the CTE difference with respect to the substrate. These results suggest that the strain energy, proportional to the square of $\varepsilon$, promotes the lateral growth of Ge crystals. This behavior is consistent with the reports on strain-induced growth promotion in amorphous $\mathrm{Si}$ and Ge layers ${ }^{14,32,33}$.

We analyze the influences of the strain on the electrical properties of the Ge layer using Hall effect measurements at $300 \mathrm{~K}$ and $115 \mathrm{~K}$. Figure 5 (a) shows that all samples exhibit hole concentrations $p$ on the order of $10^{17} \mathrm{~cm}^{-3}$ owing to defect-induced acceptors ${ }^{23,24}$. The STO sample has a relatively high $p$. Its origin is unclear. It could be attributed to the oxygen degassing from the STO substrate ${ }^{34}$, which may slightly damage the Ge layer. $p$ is decreased for all samples at $115 \mathrm{~K}$, which is a general semiconductor behavior owing to the inactivation of acceptors $^{24}$. Figure 5(b) shows that $\mu$ does not have a clear tendency with respect to $\Delta \alpha$. According to the relationship between $\mu$ and $p$ for single-crystal $\mathrm{Ge}^{1}, \mu$ of the studied Ge layers is limited by both impurity and GB carrier 
scatterings ${ }^{35} . \mu$ is increased for all samples at $115 \mathrm{~K}$, which reflects the reduction in $p$, i.e., the impurity carrier scattering. This behavior is opposite to that of common polycrystalline semiconductors, where $\mu$ is limited only by GB scattering ${ }^{35}$. We define $\mu$ increase rate $R_{\mu}$ by

$$
R_{\mu}=\frac{\mu_{115}-\mu_{300}}{\mu_{300}},
$$

where the subscripts indicate the measurement temperatures ( $300 \mathrm{~K}$ and $115 \mathrm{~K}$ ). Figure 5 (b) shows that $R_{\mu}$ increases with $\Delta \alpha$. We estimate the GB barrier height $E_{\mathrm{B}}$ by the conduction model for polycrystalline semiconductors proposed by Seto ${ }^{35}$. According to this model, $\mu$ limited by GB scattering can be determined by

$$
\mu=\frac{L q}{\sqrt{2 \pi m^{*} k T}} \exp \left(-\frac{E_{B}}{k T}\right),
$$

where $T$ is the absolute temperature, $L$ is the grain size, $m^{*}$ is the effective mass, and $k$ is the Boltzmann constant. Figure 5(c) shows that $\mu T^{1 / 2}$ increases with $1000 / T$, and then begins to decrease. This behavior indicates that $\mu$ is affected by the impurity scattering near room temperature and limited by GB scattering at low temperatures. In addition, the shapes of the Arrhenius plots significantly differ for the different substrates, which indicates a large difference in $E_{\mathrm{B}}$. Figure $5(\mathrm{~d})$ shows that $E_{\mathrm{B}}$ decreases with the increase in $\Delta \alpha$. The mechanism leading to the $E_{\mathrm{B}}$ behavior remains unclear, but it may possibly be due to the piezoelectric effect, which can occur in most polycrystalline materials ${ }^{36}$. The $E_{\mathrm{B}}$ behavior is responsible for the $R_{\mu}$ behavior in Fig. 5(b), i.e., the Ge layer with a larger $\Delta \alpha$ has a larger influence of the impurity scattering on $\mu$ because of the smaller $E_{\mathrm{B}}$. Even though the $\mathrm{GeO}_{2}$ buffer layer is provided, it is impossible to extract only the effect of strain while using substrates of different materials. However, it was strongly suggested that the substrate that provides compressive strain is better at lowering the $E_{\mathrm{B}}$ of poly-Ge.

\section{Conclusions}

We modulated the strain on poly-Ge formed by SPC at $375^{\circ} \mathrm{C}$ using various substrates, which influenced both crystal growth and electrical properties. The strain of poly-Ge was in the range of approximately $0.5 \%$ (tensile) to $-0.5 \%$ (compressive), which reflected the thermal expansion difference with respect to the substrate and phase transition of $\mathrm{Ge}$ from amorphous to crystalline. For both tensile and compressive strains, larger strains produced larger crystal grains, which reached sizes of approximately $10 \mu \mathrm{m}$. $E_{\mathrm{B}}$ was strongly dependent on the strain direction. The tensile strain increased $E_{\mathrm{B}}$, while the compressive strain reduced $E_{\mathrm{B}}$. These findings will be useful for the design of IoT devices based on poly-Ge layers on various materials.

Received: 30 September 2020; Accepted: 24 March 2021

Published online: 15 April 2021

\section{References}

1. Sze, S. M. \& Irvin, J. C. Resistivity, mobility and impurity levels in GaAs, Ge, and Si at 300 degrees. Solid. State. Electron. 11, 599-602 (1968).

2. Germain, P., Zellama, K., Squelard, S., Bourgoin, J. C. \& Gheorghiu, A. Crystallization in amorphous germanium. J. Appl. Phys. 50, 6986-6994 (1979).

3. Takagi, S. et al. III-V/Ge channel MOS device technologies in nano CMOS era. Jpn. J. Appl. Phys. 54, 06FA01 (2015).

4. Mizubayashi, W. et al. Impacts of plasma-induced damage due to UV light irradiation during etching on Ge fin fabrication and device performance of Ge fin field-effect transistors. Appl. Phys. Express 10, 026501 (2017).

5. Toriumi, A. \& Nishimura, T. Germanium CMOS potential from material and process perspectives: Be more positive about germanium. Jpn. J. Appl. Phys. 57, 010101 (2018).

6. Feng, J. et al. High-performance gate-all-around GeOI p-MOSFETs fabricated by rapid melt growth using plasma nitridation and $\mathrm{ALD} \mathrm{Al} \mathrm{O}_{3}$ gate dielectric and self-aligned NiGe contacts. IEEE Electron Device Lett. 29, 805-807 (2008).

7. Hosoi, T., Suzuki, Y., Shimura, T. \& Watanabe, H. Mobility characterization of Ge-on-insulator metal-oxide-semiconductor fieldeffect transistors with striped Ge channels fabricated by lateral liquid-phase epitaxy. Appl. Phys. Lett. 105, 173502 (2014).

8. Sadoh, T., Kamizuru, H., Kenjo, A. \& Miyao, M. Low-temperature formation $\left(<500^{\circ} \mathrm{C}\right)$ of poly-Ge thin-film transistor with $\mathrm{NiGe}$ Schottky source/drain. Appl. Phys. Lett. 89, 192114 (2006).

9. Toko, K., Nakao, I., Sadoh, T., Noguchi, T. \& Miyao, M. Electrical properties of poly-Ge on glass substrate grown by two-step solid-phase crystallization. Solid. State. Electron. 53, 1159-1164 (2009).

10. Hara, A., Nishimura, Y. \& Ohsawa, H. Self-aligned metal double-gate junctionless p-channel low-temperature polycrystallinegermanium thin-film transistor with thin germanium film on glass substrate. Jpn. J. Appl. Phys. 56, 03BB01 (2017).

11. Kabuyanagi, S., Nishimura, T., Nagashio, K. \& Toriumi, A. Impacts of oxygen passivation on poly-crystalline germanium thin film transistor. Thin Solid Films 557, 334-337 (2014).

12. Kasirajan, H. A. et al. $\mathrm{CO}_{2}$ laser annealing of low-hole-concentration polycrystalline germanium for the fabrication of enhancement-mode nMOSFET. Appl. Phys. Express 11, 101305 (2018).

13. Asadirad, M. et al. High-performance flexible thin-film transistors based on single-crystal-like germanium on glass. Adv. Electron. Mater. 2, 1600041 (2016).

14. Hekmatshoar, B., Mohajerzadeh, S., Shahrjerdi, D. \& Robertson, M. D. Thin-film tunneling transistors on flexible plastic substrates based on stress-assisted lateral growth of polycrystalline germanium. Appl. Phys. Lett. 85, 1054-1056 (2004).

15. Kasahara, K. et al. Electrical properties of pseudo-single-crystalline germanium thin-film-transistors fabricated on glass substrates. Appl. Phys. Lett. 107, 142102 (2015).

16. Suzuki, T., Joseph, B. M., Fukai, M., Kamiko, M. \& Kyuno, K. Low-temperature $\left(330^{\circ} \mathrm{C}\right)$ crystallization and dopant activation of Ge thin films via AgSb-induced layer exchange: Operation of an n-channel polycrystalline Ge thin-film transistor. Appl. Phys. Express 10, 95502 (2017).

17. Toko, K., Yoshimine, R., Moto, K. \& Suemasu, T. High-hole mobility polycrystalline Ge on an insulator formed by controlling precursor atomic density for solid-phase crystallization. Sci. Rep. 7, 16981 (2017). 
18. Takeuchi, W. et al. High hole mobility tin-doped polycrystalline germanium layers formed on insulating substrates by lowtemperature solid-phase crystallization. Appl. Phys. Lett. 107, 22103 (2015).

19. Sadoh, T., Kai, Y., Matsumura, R., Moto, K. \& Miyao, M. High carrier mobility of Sn-doped polycrystalline-Ge films on insulators by thickness-dependent low-temperature solid-phase crystallization. Appl. Phys. Lett. 109, 232106 (2016).

20. Moto, K., Yoshimine, R., Suemasu, T. \& Toko, K. Improving carrier mobility of polycrystalline Ge by Sn doping. Sci. Rep. 8, 14832 (2018).

21. Moto, K. et al. Polycrystalline thin-film transistors fabricated on high-mobility solid-phase-crystallized Ge on glass. Appl. Phys. Lett. 114, 212107 (2019).

22. Imajo, T., Moto, K., Yoshimine, R., Suemasu, T. \& Toko, K. High hole mobility ( $\left.\geq 500 \mathrm{~cm}^{2} \mathrm{~V}^{-1} \mathrm{~s}^{-1}\right)$ polycrystalline Ge films on $\mathrm{GeO}_{2}$-coated glass and plastic substrates. Appl. Phys. Express 12, 015508 (2019).

23. Haesslein, H., Sielemann, R. \& Zistl, C. Vacancies and self-interstitials in germanium observed by perturbed angular correlation spectroscopy. Phys. Rev. Lett. 80, 2626-2629 (1998).

24. Yang, H., Wang, D. \& Nakashima, H. Evidence for existence of deep acceptor levels in SiGe-on-insulator substrate fabricated using Ge condensation technique. Appl. Phys. Lett. 95,122103 (2009).

25. Takahara, D., Moto, K., Imajo, T., Suemasu, T. \& Toko, K. Sb-doped crystallization of densified precursor for n-type polycrystalline Ge on an insulator with high carrier mobility. Appl. Phys. Lett. 114, 082105 (2019).

26. Saito, M., Moto, K., Nishida, T., Suemasu, T. \& Toko, K. High-electron-mobility $\left(370 \mathrm{~cm}^{2} / \mathrm{Vs}\right)$ polycrystalline Ge on an insulator formed by As-doped solid-phase crystallization. Sci. Rep. 9, 16558 (2019).

27. Taraschi, G., Pitera, A. J. \& Fitzgerald, E. A. Strained Si, SiGe, and Ge on-insulator: review of wafer bonding fabrication techniques. Solid. State. Electron. 48, 1297-1305 (2004).

28. Fischetti, M. V. \& Laux, S. E. Band structure, deformation potentials, and carrier mobility in strained Si Ge, and SiGe alloys. J. Appl. Phys. 80, 2234-2252 (1996).

29. Wolf, S \& Taubner, R. N, Silicon Processing for the VLSI Era. Vol. I: Process Technology (Lattice, Sunset Beach, 1986).

30. Manganelli, C. L. et al. Temperature dependence of strain-phonon coefficient in epitaxial Ge/Si(001): a comprehensive analysis. J. Raman Spectrosc. 51, 989-996 (2020).

31. Miura, H., Ohta, H., Okamoto, N. \& Kaga, T. Crystallization-induced stress in silicon thin films. Appl. Phys. Lett. 60, 2746-2748 (1992).

32. Moniwa, M. et al. Preferential nucleation along $\mathrm{SiO}_{2}$ steps in amorphous Si. Appl. Phys. Lett. 47, 113-115 (1985).

33. Toko, K., Sadoh, T. \& Miyao, M. Indentation-induced low-temperature solid-phase crystallization of Si1- ${ }_{x} \mathrm{Gex}(x=0-1)$ on insulator. Appl. Phys. Lett. 94, 192106 (2009).

34. Chang, Y. J. \& Phark, S. H. Atomic-scale visualization of initial growth of perovskites on $\mathrm{SrTiO}_{3}(001)$ using scanning tunneling microscope. Curr. Appl. Phys. 17, 640-656 (2017).

35. Seto, J. W. Y. The electrical properties of polycrystalline silicon films. J. Appl. Phys. 46, 5247-5254 (1975).

36. Baraki, R. et al. Varistor piezotronics: Mechanically tuned conductivity in varistors. J. Appl. Phys. 118, 085703 (2015).

\section{Acknowledgements}

This study was financially supported by a Grant-in-Aid for JSPS Research Fellow (No. 19J21034), TEPCO Memorial Foundation, and JST PRESTO (No. JPMJPR17R7). The authors are grateful to Prof. T. Sakurai (University of Tsukuba) for the Hall effect measurements and Ms. T. Tawara and Mr. K. Tanigawa (University of Tsukuba) for their help with the experiments at Nanotechnology Platform. The EBSD measurements were carried out at the International Center for Young Scientists in NIMS.

\section{Author contributions}

K.T. and T.I. conceived and designed the experiments. T.I. conducted the experiments and analyses. K.T. and T.S. managed the research and supervised the project. All the authors discussed the results and commented on the manuscript.

\section{Competing interests}

The authors declare no competing interests.

\section{Additional information}

Correspondence and requests for materials should be addressed to T.I. or K.T.

Reprints and permissions information is available at www.nature.com/reprints.

Publisher's note Springer Nature remains neutral with regard to jurisdictional claims in published maps and institutional affiliations.

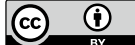

Open Access This article is licensed under a Creative Commons Attribution 4.0 International License, which permits use, sharing, adaptation, distribution and reproduction in any medium or format, as long as you give appropriate credit to the original author(s) and the source, provide a link to the Creative Commons licence, and indicate if changes were made. The images or other third party material in this article are included in the article's Creative Commons licence, unless indicated otherwise in a credit line to the material. If material is not included in the article's Creative Commons licence and your intended use is not permitted by statutory regulation or exceeds the permitted use, you will need to obtain permission directly from the copyright holder. To view a copy of this licence, visit http://creativecommons.org/licenses/by/4.0/.

(C) The Author(s) 2021 